\title{
Polyelectrolyte Self-assembled Modified Ceramic Membrane and its Application in Lithium-Sulfur Batteries
}

Ying Wang ${ }^{1 \#}$, Xue $\mathrm{Li}^{1 \#}$, Xiuqiong $\mathrm{Hu}^{2}$, Yun $\mathrm{An}^{2}$, Zhingcong $\mathrm{Ni}^{1}$, Hongyu Cheng ${ }^{3}$, Yanjie Wang ${ }^{1}$,
Zhengwu Yang ${ }^{2}$, Yuan Zhang ${ }^{1}$, Yiyong Zhang ${ }^{1, *}$, Yingjie Zhang ${ }^{1, *}$

${ }^{1}$ National and Local Joint Engineering Laboratory for Lithium-ion Batteries and Materials Preparation Technology, Faculty of Materials Science and Engineering, Faculty of Metallurgical and Energy Engineering, Kunming University of Science and Technology, Kunming 650093, China

${ }^{2}$ College of Intelligent Manufacture, PanZhihua University, PanZhihua 617000, China

${ }^{3}$ Faculty of Materials Science and Engineering, Kunming University of Science and Technology, Kun ming 650093, China.

*E-mail: zhangyiyong0918@qq.com, zhangyingjie09@126.com

\#Ying Wang and Xue Li contributed equally to this work as co-first authors.

doi: $10.20964 / 2020.12 .02$

Received: 31 July 2020 / Accepted: 17 September 2020 / Published: 31 October 2020

As one of the candidates for lithium-ion batteries, lithium-sulfur ( $\mathrm{Li}-\mathrm{S})$ batteries have received extensive research and attention. However, the shuttle effect of soluble lithium polysulfide has hindered the commercial application of Li-S batteries. Therefore, this paper proposes the use of a polyelectrolyte selfassembled modified ceramic separator to inhibit the shuttling of lithium polysulfide intermediates. First, a layer of silica was applied on the surface of a polyethylene (PE) separator. Then, polyethyleneimine (PEI), polyacrylic acid (PAA), polydiallyldimethylammonium chloride (PDDA) and sodium polystyrene sulfonate (PSSNa) were selected to synthesize two self-assembled cationic/anionic polyelectrolytes. Finally, layer-by-layer (LBL) self-assembly technology was used to grow the polyelectrolyte in situ on the ceramic separator to form a functional separator. Galvanostatic charge/discharge revealed that the LBL4-PEI-PAA separator and LBL4-PDDA-PSSNa separator have improved specific capacity and cycle performance compared to the $\mathrm{PE}$ separator, and there is no obvious overcharge phenomenon when using an electrolyte without a lithium nitrate additive. In particular, the LBL4-PDDA-PSSNa separator has a first-lap discharge specific capacity of $981.8 \mathrm{mAh} / \mathrm{g}$. The capacity retention rate is $88.9 \%$ after 100 cycles, which effectively suppresses the lithium polysulfide shuttle effect.

Keywords: lithium-sulfur battery, shuttle effect, self-assembly, cation/anionic polyelectrolytes 
(C) 2021 The Authors. Published by ESG (www.electrochemsci.org). This article is an open access article distributed under the terms and conditions of the Creative Commons Attribution license (http://creativecommons.org/licenses/by/4.0/). 